\section{Acute kidney injury associated with foot osteomyelitis}

The occurrence of acute kidney injury (AKI) associated with foot osteomyelitis has been reported infrequently and only in case report format. ${ }^{1-3}$ After noting the frequent occurrence of AKI among patients presenting with foot osteomyelitis at our institution, we endeavoured to estimate the prevalence rate and identify associated factors.

After obtaining institutional review board approval, we reviewed the electronic medical records of all patients presenting to the Michael E DeBakey Veterans Affairs Medical Center with an initial episode of 'probable' or 'definite' foot osteomyelitis ${ }^{4}$ between 1 January 2011 and 1 March 2015. Patients with preexisting end-stage renal disease were excluded. During this time period, all patients with foot osteomyelitis were managed by a surgical team using a local treatment algorithm; the operative logs and clinical registry of this team served to identify patients meeting inclusion/ exclusion criteria. AKI was defined as an increase in serum creatinine by $0.3 \mathrm{mg} / \mathrm{dL}$ or greater within 48 hours of admission or a serum creatinine $\geq 1.5$ times than baseline that is known or presumed to have occurred within the last 7 days. ${ }^{5}$ Baseline variables recorded included age, estimated glomerular filtration rate (as per modified Crockcoft-Gault), presence of micro- or macro-albuminuria, signs of systemic inflammatory response syndrome and bacterial isolates identified.

In total, 173 patients met inclusion criteria. The median age was 64 years. Diabetes mellitus was present in 155 patients (89.6\%). Stage 3 or higher chronic kidney disease was present in 62 patients $(35.8 \%)$. A concomitant soft tissue abscess was present in 118 patients $(68.2 \%)$.

In total, 32 of the 173 patients (18.5\%) developed AKI. Eighteen of these patients (56.3\%) had AKI at the time of admission, while the remaining 14 patients $(43.8 \%)$ with AKI met criteria within the subsequent 48 hours after admission. The median increase in serum creatinine among those with AKI was $0.69 \mathrm{mg} / \mathrm{dL}$, a $57 \%$ increase over pre-admission baseline.

Logistic regression demonstrated that the development of AKI was inversely correlated with serum albumin (odds ratio 0.54 per $\mathrm{mg} / \mathrm{dL}, \mathrm{p}=0.03$ ). A trend toward AKI was observed with the presence of associated soft tissue abscess (odds ratio $2.2, \mathrm{p}=0.11)$. We did not find a significant association between AKI and estimated glomerular filtration rate $(p=0.71)$, microalbuminuria $(\mathrm{p}=0.80)$, macro-albuminuria $(\mathrm{p}=0.62)$, or the number of systemic inflammatory response syndrome criteria present at admission $(\mathrm{p}=0.40)$.

We suspect most clinicians managing patients with foot osteomyelitis are unaware of the association with AKI. The lack of association between AKI and intuitive risk factors such as baseline chronic kidney disease, presence of macroalbuminuria and the presence of systemic inflammatory response syndrome also makes predicting which patients are at risk more difficult. The high prevalence we are reporting has direct clinical relevance, including management of intravenous medications, selection and dosing of antimicrobial agents and minimisation/avoidance of iodinated contrast media and medications with potential nephrotoxicity.

\section{Conflicts of interest}

The authors have no conflicts of interest to declare.

\section{Author Contributions}

Research idea and study design: BCJ, JBC, NRB; data acquisition: BCJ, $\mathrm{NRB}$; data analysis/interpretation: $\mathrm{BCJ}$, JBC, NRB; statistical analysis: NRB; supervision and mentorship: JBC, NRB. All authors contributed important intellectual content during the manuscript drafting or revision and accept accountability for the overall work by ensuring that questions pertaining to the accuracy or integrity of any portion of the work are appropriately investigated and resolved.

\section{Acknowledgements}

This study is covered under the Baylor College of Medicine institutional review board protocol BCM IRB H-34858 titled Pilot study to identify factors useful in the clinical management of diabetic foot osteomyelitis.

BRYAN C JIANG Clinical fellow in endocrinology, diabetes and metabolism, Baylor College of Medicine, Houston, Texas, USA

JeNNIFER B COWART Assistant professor of medicine, The Mayo Clinic, Jacksonville, Florida, USA

NeAL R BARSHES Associate professor of surgery, Division of Vascular and Endovascular Surgery, Michael E. Debakey Department of Surgery, Baylor College of Medicine / Michael E. DeBakey Veterans Affairs Medical Center, Houston, Texas, USA

\section{References}

1 Spital A, Sterns RH, Panner BJ. Glomerulonephritis and reversible renal failure resulting from osteomyelitis in a diabetic patient. Am J Med 1988;85:235-6.

2 Griffin MD, Björnsson J, Erickson SB. Diffuse proliferative glomerulonephritis and acute renal failure associated with acute staphylococcal osteomyelitis. J Am Soc Nephrol JASN 1997;8:1633-9.

3 Tevlin MT, Wall BM, Cooke CR. Reversible renal failure due to IgA nephropathy associated with osteomyelitis. Am J Kidney Dis Off J Natl Kidney Found 1992;20:185-8.

4 Berendt AR, Peters EJG, Bakker K et al. Diabetic foot osteomyelitis: a progress report on diagnosis and a systematic review of treatment. Diabetes Metab Res Rev 2008;24(Suppl 1):S145-61.

5 Kellum JA, Lameire N, KDIGO AKI Guideline Work Group. Diagnosis, evaluation, and management of acute kidney injury: a KDIGO summary (Part 1). Crit Care 2013;17:204.

\section{Testing for blood-borne viruses after a needle-stick} injury in patients who lack the capacity to consent

A conclusion that a person lacks decision making capacity is not an off-switch for his or her rights ${ }^{1}$

It is usual practice after a needle-stick injury for the source patient, with consent, to be tested for blood-borne viruses (BBV) to guide the need for HIV prophylaxis and to organise appropriate follow-up of the recipient. If the source patient cannot give consent and therefore is not tested then this uncertainty can heighten the injury-associated anxiety and result in unnecessary prophylaxis for the recipient. General Medical Council (GMC) guidance states that BBV testing for the sole benefit of a healthcare worker is unlawful and may only be performed if it is in the best interests of the patient. ${ }^{2}$ The GMC, however, does not clearly define the best interests of the patient in this scenario, so the 
Box 1. A guide to testing

1 Does the patient have capacity?

2 Is the lack of capacity likely to be temporary?*

3 Has the patient recently been tested for blood-borne viruses? A thorough search of both primary and secondary care records is necessary before proceeding further.

4 If prolonged lack of capacity, does the patient have an attorney or a deputy with the legal authority to make decisions for him or her?**

5 If there is no legal deputy then speak to friends and family to establish whether the patient would have ordinarily objected to being tested for this purpose?

6 If there is no recent test result available and there is no evidence that the patient would have ordinarily objected then testing can proceed

7 To minimise harm, testing should be performed on a recently stored sample or if a new sample is needed it should be taken at the same time as blood is taken for other clinical purposes.

8 Once the patient regains capacity they should be advised of the needle-stick injury and any testing that was undertaken and appropriate information should be provided so that the patient can make an informed decision about whether to receive the result.

9 It should be accepted that some patients may not want to know the results but it must be remembered that consent is an ongoing process and a patient who declines to receive the result at a certain point may decide otherwise in the future and adequate support should be put into place so that a change in consent can be accommodated.

"There is no specific time frame for 'temporary' but a patient undergoing anaesthetic for a surgical procedure should be considered in this category and in other situations clinical judgement should be exercised.

**Being next of kin alone does not confer legal power for decision making.

British Medical Association (BMA) issued guidance in 2016 to fill this gap. ${ }^{3}$ This letter sets out to summarise the ethical arguments that underpin the BMA guidance, illustrate how this guidance could be put into practice and raise debate on this issue.

There are two main strands to the guidance, the first focuses on clinical best interest. It may be argued that for most individuals it is in their best interest to know whether they have a BBV as they are likely to benefit from current or future care of that infection. Often the question asked by the clinician at the time of such an incident is 'will it influence current management'; however, the guidance encourages the clinician to think more broadly and holistically, which makes the question of the timeliness of testing redundant.

The second strand focuses on the broader best interest of an individual, which encompasses a much more person-centred approach. The limited evidence available suggests that only the minority of patients do not give consent for BBV testing when they do have capacity. ${ }^{4}$ So, why might an individual be tested primarily for the interests of another? There are several ethical imperatives that may explain such altruism and these include altruism in itself, reciprocity of care and recognition of the importance of the relationship between the patient and healthcare worker, for the greater good of all patients as all patients would benefit from the health professional being appropriately treated and being able to continue working and fulfilling the duties of the responsible citizen.

The guidance invites the user to construct a balance sheet of benefits and dis-benefits of testing, diligently following the steps listed in Box 1. In essence, the BMA guidance highlights that testing that benefits a third party and patient best interest are not mutually exclusive and that patients who lack capacity must not have their best interests neglected.

\section{Conflicts of interest}

The author has no conflicts of interest.

RAJEKA LAZARUS

Registrar in infectious diseases and microbiology, Oxford University Hospital Foundation Trust, Oxford, UK

\section{References}

1 Wye Valley NHS Trust v B [2015]EWCOP 60.

2 General Medical Council. Consent: patients and doctors making decisions together. London: GMC, 2008.

3 British Medical Association. Needlestick injuries and blood-borne viruses: decisions about testing adults who lack the capacity to consent. London: BMA, 2016.

4 Giri P, Basu S, Adisesh A, Rimmer A. Blood and body fluid exposures: consent for source patient testing. Occup Med 2013;63:135-7.

\section{Sodium glucose co-transporter 2 inhibitors successfully attenuated seasonal change of glycated haemoglobin A1c}

Previous studies suggested that glycated haemoglobin (HbAlc) levels are highest in winter and lowest in summer. ${ }^{1-4}$ Potential susceptibilities to seasonal change of HbAlc include inappropriate dietary calorie intake and insufficient physical activity in winter. In our hospital, the majority of diabetes patients are farmers and their physical activity is relatively lower in winter (agricultural off-season) than in summer (busy farming season). These patients face difficulties in adapting a seasonal diet modification concomitant with the variation in physical activity.

In this study, we examined the effect of sodium glucose co-transporter 2 (SGLT2) inhibitors on patients who experience seasonal change of their diabetic control. Because those patients also tend to increase body weight, SGLT2 inhibitors were considered as an addition to their current medications to minimise seasonal variation of HbAlc in winter. Our study protocol was reviewed and approved by our hospitals' review boards according to the Declaration of Helsinki. Written informed consent was obtained from each participant. This clinical study involved 30 patients with type 2 diabetes mellitus whose HbAlc showed seasonal change for the previous 3 years and got worse in the autumn of 2015. Patients were prescribed an SGLT2 inhibitor in addition to their current medications. Patients visited the hospital for follow-up examination once a month, and blood glucose levels and HbAlc levels from the same blood samples were measured. In parallel, urinary glucose levels were determined in spot urine samples at each visit. Body 